\title{
On the Trigonometric Loophole
}

\author{
Jiř́i Stávek ${ }^{1}$ \\ ${ }^{1}$ Prague, Czech Republic \\ Correspondence: Jiř́i Stávek, Bazovského 1228, Prague 163 00, Czech Republic. E-mail: stavek.jiri@seznam.cz
}

Received: August 13, 2013 Accepted: September 2, 2013 Online Published: October 18, 2013

doi:10.5539/apr.v5n6p48

URL: http://dx.doi.org/10.5539/apr.v5n6p48

\begin{abstract}
Two free-will partners A (Alice) and B (Bob) share each a photon from a photon pair emitted from two mutually perpendicularly adjacent non-linear crystals (the spontaneous parametric downconversion SPDC Type I). The third partner $C$ (Claudius) discovered two local hidden trigonometric functions: Cosag $(2 \theta)=$ Cosagitta $(2 \theta)=$ $\cos ^{2}(\theta)=\downarrow$ and Sag $(2 \theta)=$ Sagitta $(2 \theta)=\sin ^{2}(\theta)=\uparrow$ that govern the geometrical (local) probability of correlation and anti-correlation outcomes, respectively. Cosagitta $(2 \theta)=\cos ^{2}(\theta)$ and Sagitta $(2 \theta)=\sin ^{2}(\theta)$ are the heights of an arc in the circle with diameter $\mathrm{D}=1$. The arc length distance $\mathrm{L}=( \pm \theta+\gamma)^{*} \mathrm{D}=( \pm \alpha \pm \beta+$ $\gamma)^{*} \mathrm{D}(\mathrm{D}=1)$ describes the arc length between the polarization planes of those correlated photons. The angles $\alpha$, $\beta$ and $\gamma / 2$ are the angle adjustments of three half-wave plates (HWP) to achieve the tailor-made polarization of those correlated photons. The partner C employs the Claudius' Theorem to evaluate the joint outcomes found experimentally by the partners A and B. This "trigonometric loophole" can be used to describe the observed and predicted outcomes of correlated particles.
\end{abstract}

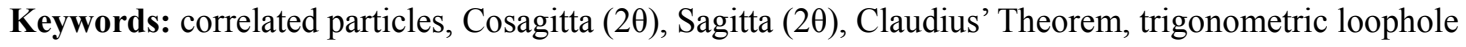

\section{Introduction}

We will celebrate 50 years since the publication of the seminal paper of John S. Bell "On the Einstein-Podolsky-Rosen Paradox" in the year 2014. Bell inequalities determine the boundary between the local-hidden-variable theories (LHVT) and the predictions of the quantum mechanics (e.g., Cabello, 2007; Fine, 1982; Genovese, 2005; Laloë, 2012). Many groups of researchers experimentally tested those correlation statistics and confirmed the predictions of the quantum mechanics.

The first generation of experimental tests of Bell's inequalities with pairs of entangled photons was carried out between the years 1970-1978 and used "single-channel" polarizers (Clauser, Horne, Shimony, \& Holt, 1969; Clauser \& Horne, 1974; Clauser \& Shimony, 1978; Freedman \& Clauser, 1972; Fry \& Thomson, 1976). The second generation of experiments between the years 1978-1990 worked with improved source of entangled photons and used the CH74 inequality and the CHSH inequality (Aspect, Dalibard \& Roger, 1982; Aspect, Grangier \& Roger, 1982; Marshall, Santos, \& Selleri, 1983; Ou \& Mandel, 1988). Experiments of the third generation in the years 1991-2000 produced the correlated photons in nonlinear spontaneous parametric downconversion (SPDC) (Kwiat, Mattle, Weinfurter, \& Zeilinger, 1995; Kwiat, Waks, White, Appelbaum, \& Eberhard, 1999; White, James, Eberhard, \& Kwiat, 1999; Zeilinger, 1999). These experiments closed the "locality loophole" (Weihs, Jennewein, Simon, Weinfurter, \& Zeilinger, 1998). It was found that the distance did not destroy entanglement (Tapster, Rarity, \& Owens, 1994; Tittel, Brendel, Zbinden, \& Gisin, 1998). Experiments of the fourth generation in the years 2001-2010 worked with significantly improved all optical elements used for those experiments (e.g., Rangarajan, Goggin, \& Kwiat, 2009; Rowe et al., 2001). Measurements were done for longer and longer distances and the entanglement was not destroyed (e.g., Ursin et al., 2007; Scheidl et al., 2009). The delayed choice entanglement was realized. Experiments of the fifth generation in the years 2011-2013 successfully closed three loopholes: "the locality loophole", "the detection loophole" and "the freedom-of-choice loophole" (e.g., Christensen et al., 2013; Giustina et al., 2013; Scheidl et al., 2010; Wittmann et al., 2013).

It seems that we have achieved "the loophole-free stage" of the Bell's theorem and that there is no hope to return back to any possible local concept for the description of outcomes of correlated particles.

The aim of this contribution is to revisit the ancient and medieval mathematical literature and to reappear with forgotten mathematical tools. Are there any powerful mathematical tools that could be helpful in the solving of 
the conundrum of the correlation statistics of entangled particles? Can we find "a trigonometric loophole"?

\section{The Forgotten Trigonometric Functions}

The development of trigonometry crossed most major cultures and places where many mathematicians contributed significantly to this topic. Several monographs on the history of trigonometry were written and can guide us on this way: Von Braumühl (1900), Datta and Singh (1983), Matvieskaya (1990), Maor (1998) and Van Brummelen $(2009,2013)$. The table of chord values was published by Claudius Ptolemy.

There are two groups of trigonometric functions - the first (ancient and medieval) group is based on the arc of a circle, the second (modern) one is based on a plane right triangle in the unit circle (Radius $\mathrm{R}=1$ ). The original sine function developed in India, unlike the modern one, was not a ratio, but simply the length of the side opposite the angle in a right triangle of fixed hypotenuse. When the sine function is a ratio instead of the length of a line segment, the straightforward relation between the length of the arc and the value of sine function disappears. Kupková (2008) compared the trigonometric functions chord and sine and stimulated our interest in the old arc trigonometric functions.

Table 1 summarizes the forgotten arc trigonometric functions for the unit circle (Radius $\mathrm{R}=1$, central angle $2 \theta$, arc length equals $2 \theta^{*} \mathrm{R}=2 \theta$ ) and for the circle with the Diameter $\mathrm{D}=1$ (central angle $2 \theta$, arc length equals $2 \theta * \mathrm{D} / 2=\theta$ ). We have chosen the central angle $2 \theta$ because we will get convenient relations for the action of the half-wave plates (HWP) on the correlated photons created by the SPDC Type I.

Table 1. The forgotten arc trigonometric functions

\begin{tabular}{lll}
\hline \multicolumn{2}{l}{ Radius $\mathbf{R}=\mathbf{1}$, central angle $=\mathbf{2 \theta}$, arc length $=\mathbf{2 \theta} \mathbf{R}=\mathbf{2 \theta}$} \\
\hline chord & chord & $\operatorname{crd}(2 \theta)=2 \sin (\theta)$ \\
versed chord & verchord & $\operatorname{vercrd}(2 \theta)=2 \cos (\theta)$ \\
versed sine & versine & $\operatorname{versin}(2 \theta)=2 \sin ^{2}(\theta)=1-\cos (2 \theta)$ \\
versed cosine & vercosine & $\operatorname{vercosin}(2 \theta)=2 \cos ^{2}(\theta)=1+\cos (2 \theta)$ \\
coversed sine & coversine & $\operatorname{coversin}(2 \theta)=2 \sin ^{2}(\pi / 4-\theta)=1-\sin (2 \theta)$ \\
coversed cosine & covercosine & $\operatorname{covercosin}(2 \theta)=2 \cos ^{2}(\pi / 4-\theta)=1+\sin (2 \theta)$ \\
\hline Diameter $\mathbf{D}=\mathbf{1}$, central angle $=\mathbf{2 \theta}, \operatorname{arc~length}=\mathbf{\theta D}=\mathbf{\theta}$ \\
\hline half chord & hachord & hacrd $(2 \theta)=\sin (\theta)$ \\
half versed chord & haverchord & havercrd $(2 \theta)=\cos (\theta)$ \\
half versed sine & haversine & haversin $(2 \theta)=\sin ^{2}(\theta)=(1-\cos (2 \theta)) / 2=\mathbf{S a g i t t a}(\mathbf{2 \theta})$ \\
half versed cosine & havercosine & havercosin $(2 \theta)=\cos ^{2}(\theta)=(1+\cos (2 \theta)) / 2=\mathbf{C o s a g i t t a}(\mathbf{2 \theta})$ \\
half coversed sine & hacoversine & hacoversin $(2 \theta)=\sin ^{2}(\pi / 4-\theta)=(1-\sin (2 \theta)) / 2$ \\
half coversed cosine & hacovercosine & hacovercosin$(2 \theta)=\cos ^{2}(\pi / 4-\theta)=(1+\sin (2 \theta)) / 2$ \\
\hline
\end{tabular}

We add Figure 1 and Figure 2 to depict graphically these arc trigonometric functions as lengths in the unit circle with the Radius $\mathrm{R}=1$ and in the circle with the Diameter $\mathrm{D}=1$. The relations in those Figure 1 and Figure 2 were checked by mathematical instruments of Euclid and Descartes. 


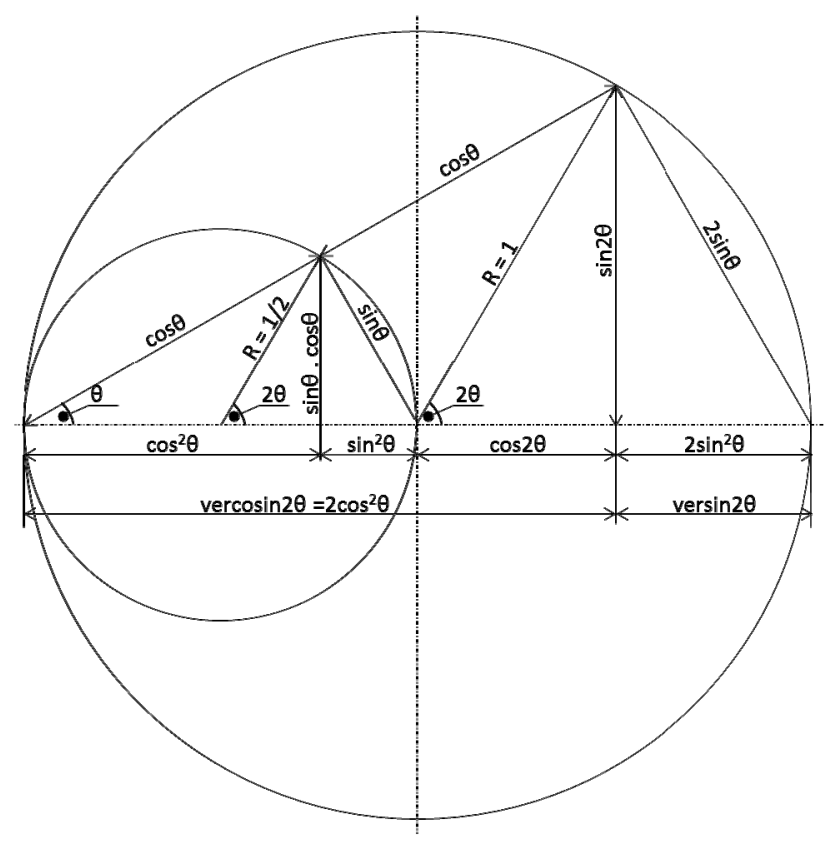

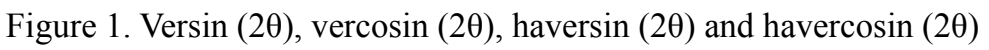

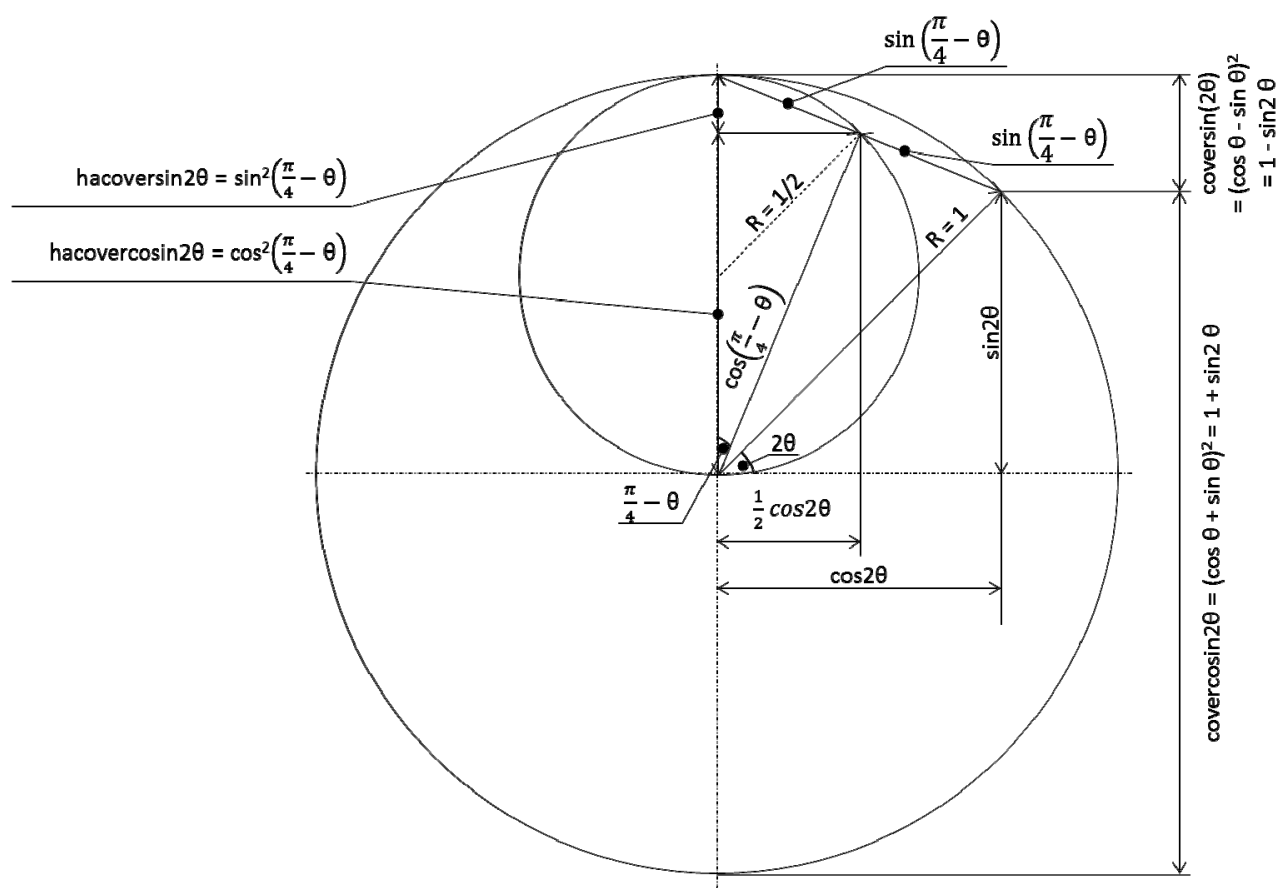

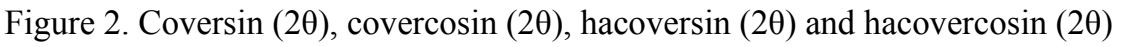

\section{Two Local Hidden Trigonometric Functions}

Ancient and medieval mathematicians had another trigonometric functions available to them than we have today. It is interesting to rediscover those forgotten trigonometric functions because some of them have very beautiful and even hidden properties.

We will use in our approach two trigonometric functions: $\operatorname{Cosag}(2 \theta)=\operatorname{Cosagitta}(2 \theta)=\cos ^{2}(\theta)=\downarrow$ and Sag $(2 \theta)$ $=$ Sagitta $(2 \theta)=\sin ^{2}(\theta)=\uparrow$ that govern the geometrical (local) probability of correlation and anti-correlation outcomes, respectively. $\operatorname{Cosag}(2 \theta)=\cos ^{2}(\theta)$ and $\operatorname{Sag}(2 \theta)=\sin ^{2}(\theta)$ are the heights of an arc in the circle with 
diameter $\mathrm{D}=1$ and the central angle $2 \theta$. The arc length distance $\mathrm{L}=( \pm \theta+\gamma) * \mathrm{D}=( \pm \alpha \pm \beta+\gamma) * \mathrm{D}(\mathrm{D}=1)$ describes the arc length between the polarization planes of those correlated photons. The angles $\alpha, \beta$ and $\gamma / 2$ are the angle adjustments of three half-wave plates (HWP) to achieve the tailor-made polarization of those correlated photons created by the spontaneous parametric downconversion-SPDC Type I.

Figure 3, Figure 4 and Figure 5 show the probabilistic outcomes of "single-clicks" at Alice's side and Bob's side, and combined probabilistic outcomes of "double clicks" calculated by Claudius. The experimental observation of these probabilities was excellently described by Alain Aspect (2000) in his conference talk "Bell's Theorem: The Naïve View of an Experimentalist".

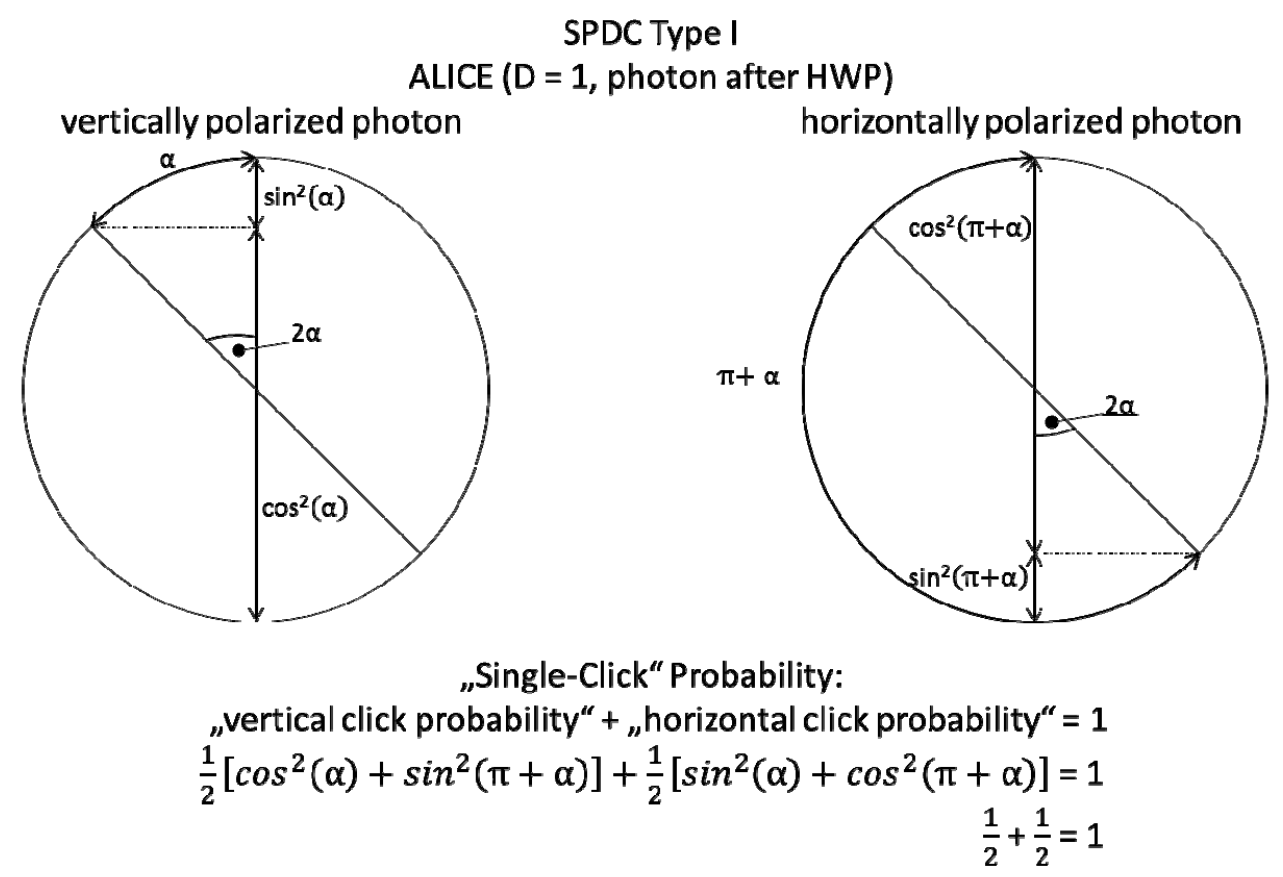

Figure 3. Probabilistic outcomes of the "single-click detection" at ALICE's side

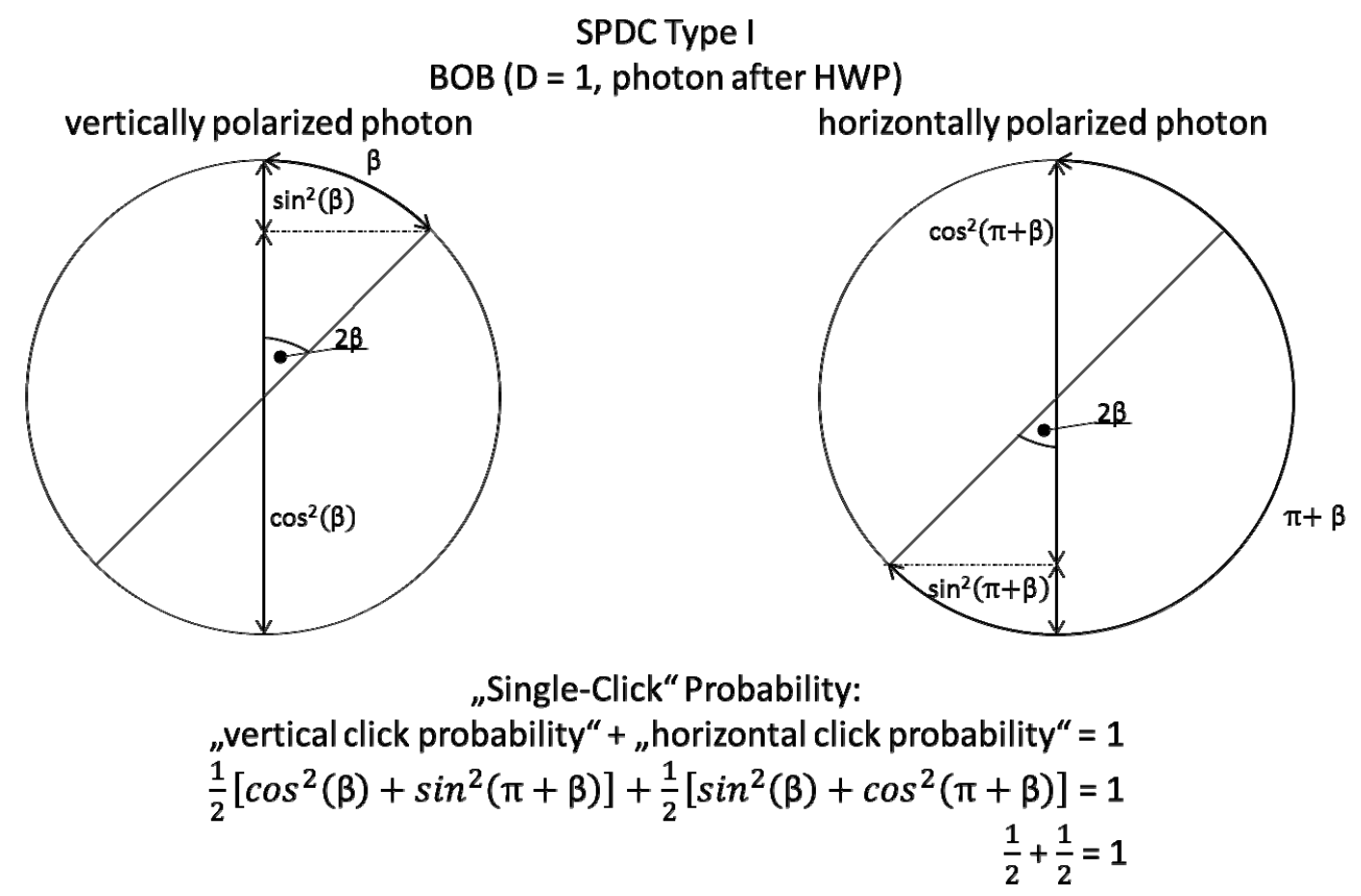

Figure 4. Probabilistic outcomes of the "single-click detection" at BOB's side 


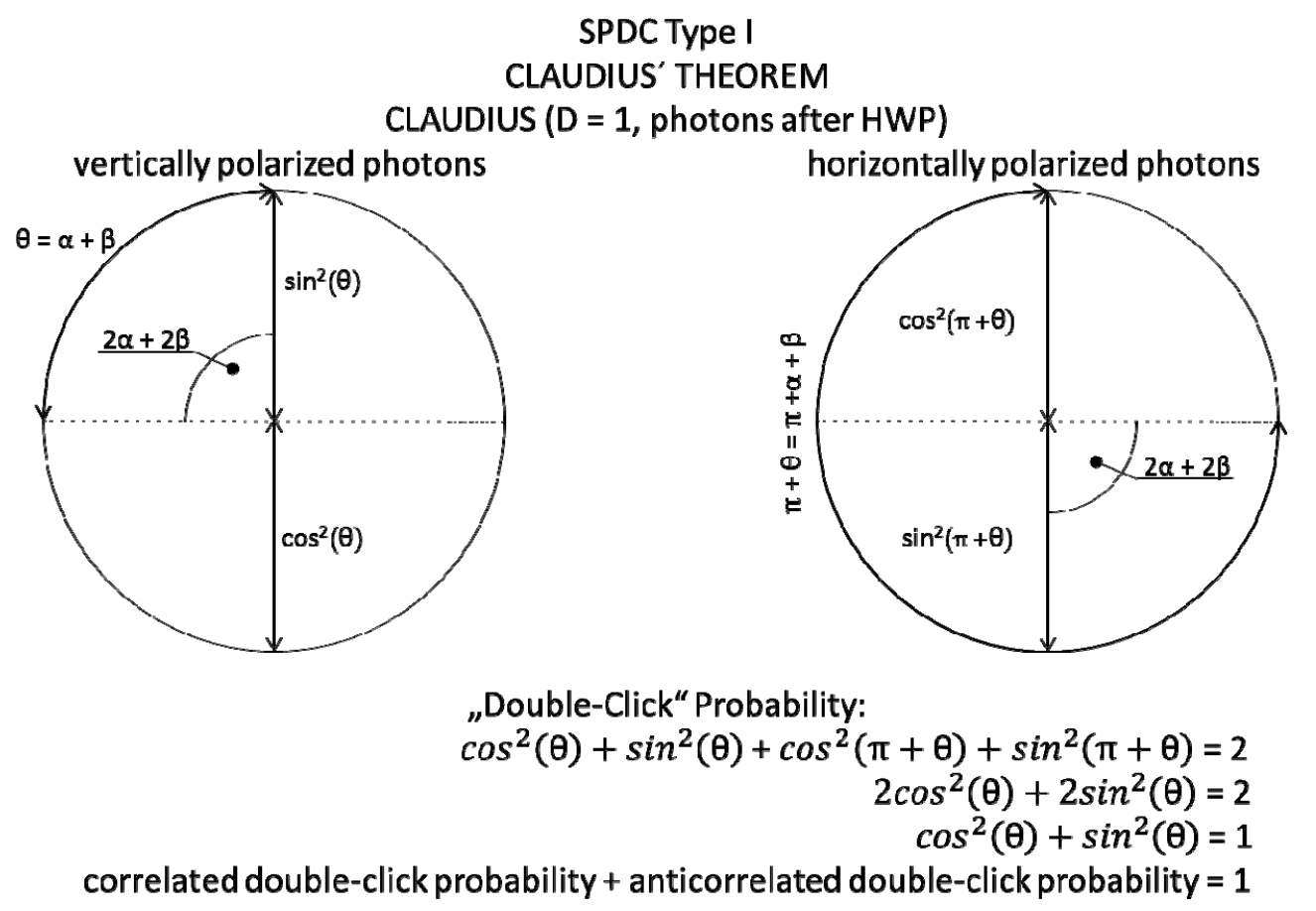

Figure 5. Probabilistic outcomes of the "double-click detection" calculated by CLAUDIUS

\section{Photon Pairs with the Tailor-Made Relative Polarization}

Correlated photon pairs can be produced with the spontaneous parametric downconversion (SPDC Type I process, Kwiat et al., 1999). In this process two mutually perpendicularly adjacent crystals create the H-polarized cone (with horizontally polarized photon pairs) and the V-polarized cone (with vertically polarized photon pairs). The photon pairs with the desired tailor-made relative polarization can be created by inserting a half-wave plate (HWP-Claudius) in either the signal beam or idler beam with the angle adjustment $\gamma / 2$. This element (HWP-Claudius) modifies the relative polarization plane distance of these two correlated photons by the arc length distance equal to $\gamma$.

Two observers Alice and Bob use their half-wave plates (HWP-Alice and HWP-Bob). Alice and Bob can freely rotate with their half-wave plates with the angle adjustment $\pm \alpha$ and $\pm \beta$. For the joint correlated or anti-correlated outcomes Claudius uses his Claudius' Theorem where he inserts the relative arc length distance L of the polarization planes of those photons where the circle diameter $\mathrm{D}=1$ :

$$
L=( \pm \alpha \pm \beta+\gamma) D=( \pm \theta+\gamma) D= \pm \theta+\gamma
$$

Claudius' Theorem calculates already observed correlation and anti-correlation outcomes (SPDC Type I and SPDC Type II) and predicts the outcomes for the arbitrary adjustments of the angle $\gamma$ (SPDC Type III, SPDC Type IV, SPDC Type VIII, etc.):

$$
\begin{aligned}
& \sin ^{2} L=\sin ^{2}(\gamma \pm \theta) \\
& \cos ^{2} L=\cos ^{2}(\gamma \pm \theta)
\end{aligned}
$$


Table 2. Correlation and anti-correlation outcomes for the SPDC Type I

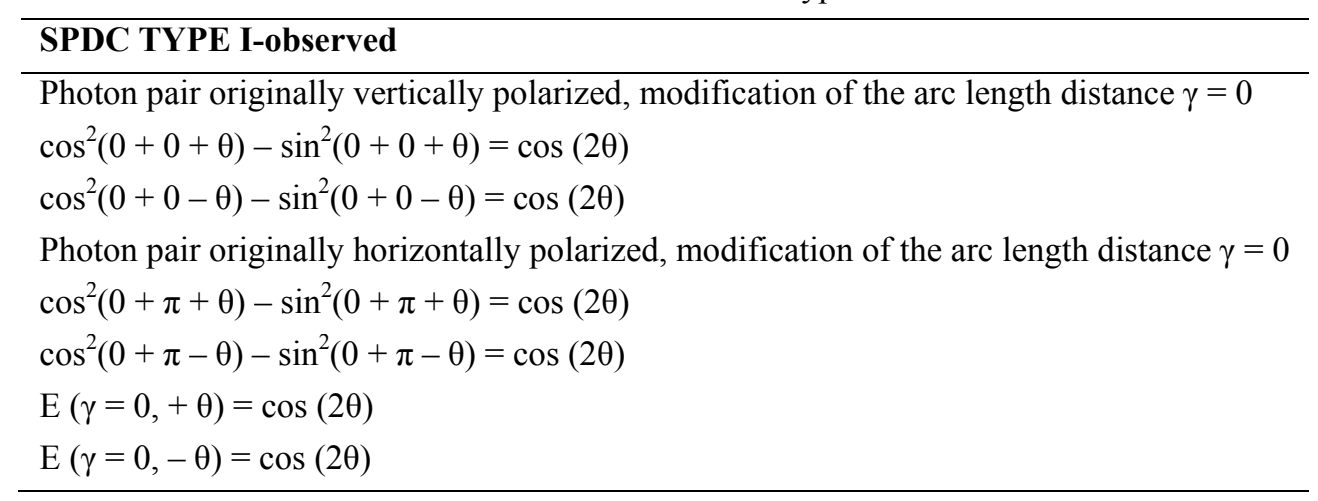

Table 3. Correlation and anti-correlation outcomes for the SPDC Type II

\section{SPDC TYPE II-observed}

Photon pair originally vertically polarized, modification of the arc length distance $\gamma=\pi / 2$

$\cos ^{2}(\pi / 2+0+\theta)-\sin ^{2}(\pi / 2+0+\theta)=-\cos (2 \theta)$

$\cos ^{2}(\pi / 2+0-\theta)-\sin ^{2}(\pi / 2+0-\theta)=-\cos (2 \theta)$

Photon pair originally horizontally polarized, modification of the arc length distance $\gamma=\pi / 2$

$\cos ^{2}(\pi / 2+\pi+\theta)-\sin ^{2}(\pi / 2+\pi+\theta)=-\cos (2 \theta)$

$\cos ^{2}(\pi / 2+\pi-\theta)-\sin ^{2}(\pi / 2+\pi-\theta)=-\cos (2 \theta)$

$\mathrm{E}(\gamma=\pi / 2,+\theta)=-\cos (2 \theta)$

$\mathrm{E}(\gamma=\pi / 2,-\theta)=-\cos (2 \theta)$

Table 4. Correlation and anti-correlation outcomes for the SPDC Type III

\section{SPDC TYPE III-predicted}

Photon pair originally vertically polarized, modification of the arc length distance $\gamma=\pi / 3$

$\cos ^{2}(\pi / 3+0+\theta)-\sin ^{2}(\pi / 3+0+\theta)=-\sin (\pi / 6+2 \theta)$

$\cos ^{2}(\pi / 3+0-\theta)-\sin ^{2}(\pi / 3+0-\theta)=-\sin (\pi / 6-2 \theta)$

Photon pair originally horizontally polarized, modification of the arc length distance $\gamma=\pi / 3$

$\cos ^{2}(\pi / 3+\pi+\theta)-\sin ^{2}(\pi / 3+\pi+\theta)=-\sin (\pi / 6+2 \theta)$

$\cos ^{2}(\pi / 3+\pi-\theta)-\sin ^{2}(\pi / 3+\pi-\theta)=-\sin (\pi / 6-2 \theta)$

$\mathrm{E}(\gamma=\pi / 3,+\theta)=-\sin (\pi / 6+2 \theta)$

$\mathrm{E}(\gamma=\pi / 3,-\theta)=-\sin (\pi / 6-2 \theta)$

Table 5. Correlation and anti-correlation outcomes for the SPDC Type IV

SPDC TYPE IV-predicted

Photon pair originally vertically polarized, modification of the arc length distance $\gamma=\pi / 4$

$\cos ^{2}(\pi / 4+0+\theta)-\sin ^{2}(\pi / 4+0+\theta)=-\sin (2 \theta)$

$\cos ^{2}(\pi / 4+0-\theta)-\sin ^{2}(\pi / 4+0-\theta)=+\sin (2 \theta)$

Photon pair originally horizontally polarized, modification of the arc length distance $\gamma=\pi / 4$

$\cos ^{2}(\pi / 4+\pi+\theta)-\sin ^{2}(\pi / 4+\pi+\theta)=-\sin (2 \theta)$

$\cos ^{2}(\pi / 4+\pi-\theta)-\sin ^{2}(\pi / 4+\pi-\theta)=+\sin (2 \theta)$

$\mathrm{E}(\gamma=\pi / 4,+\theta)=-\sin (2 \theta)$

$\mathrm{E}(\gamma=\pi / 4,-\theta)=+\sin (2 \theta)$ 
Table 6. Correlation and anti-correlation outcomes for the SPDC Type VIII

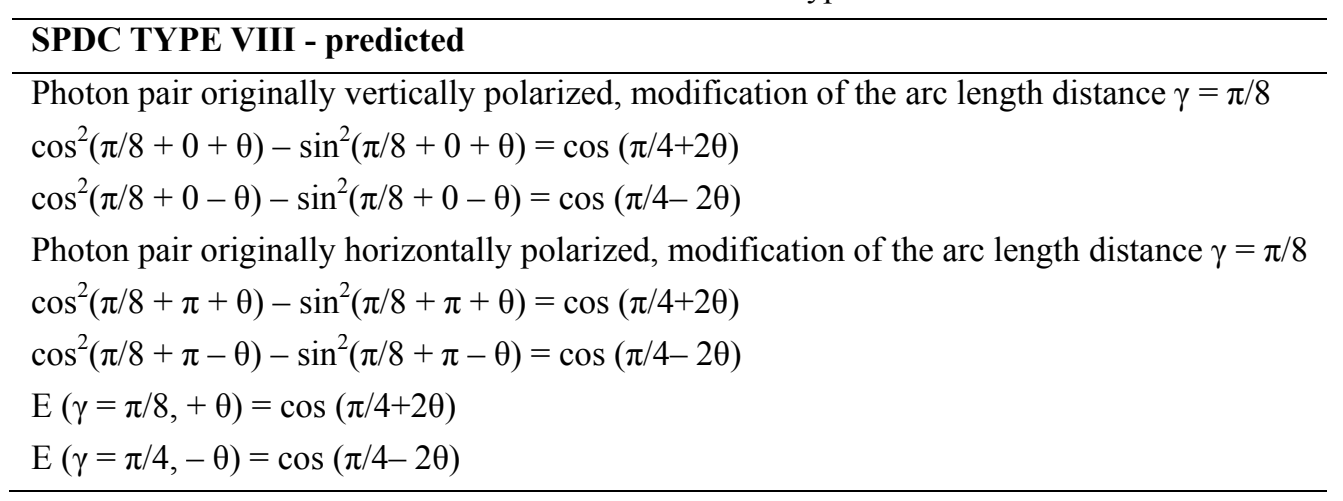

\section{Correlated Particles with Spin: 1/2, 1, 3/2, and 2}

We can apply the Claudius' Theorem for the calculation of correlation and anti-correlation outcomes for correlated particles with spin: $1 / 2,1,3 / 2$, and 2 , etc. The spin is graphically interpreted as the arc length distance of the polarization of those particles. In Figure 6 the arc length distance (spin) is influenced by the central angle and the diameter $\mathrm{D}=1$.

Table 7. Correlation and anti-correlation outcomes for particles with spin $1 / 2$

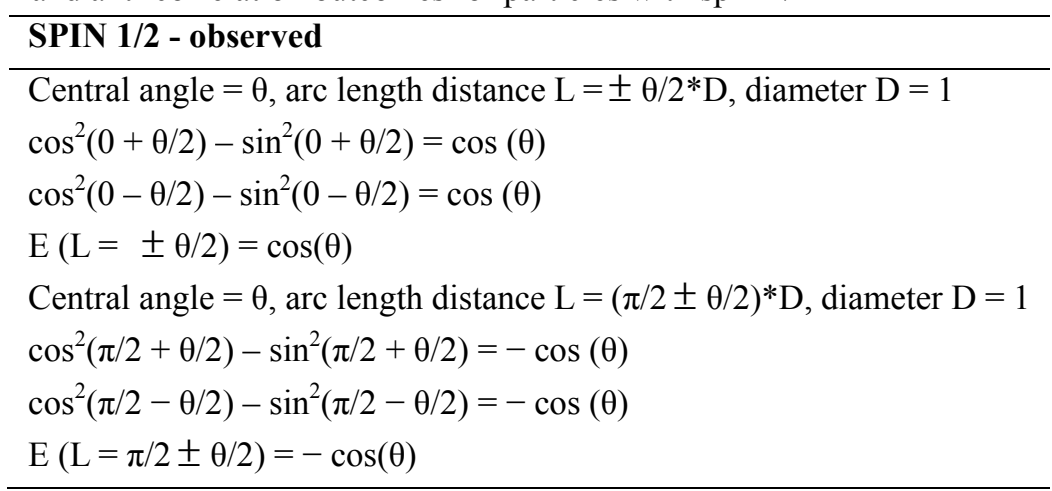

Table 8. Correlation and anti-correlation outcomes for particles with spin 1

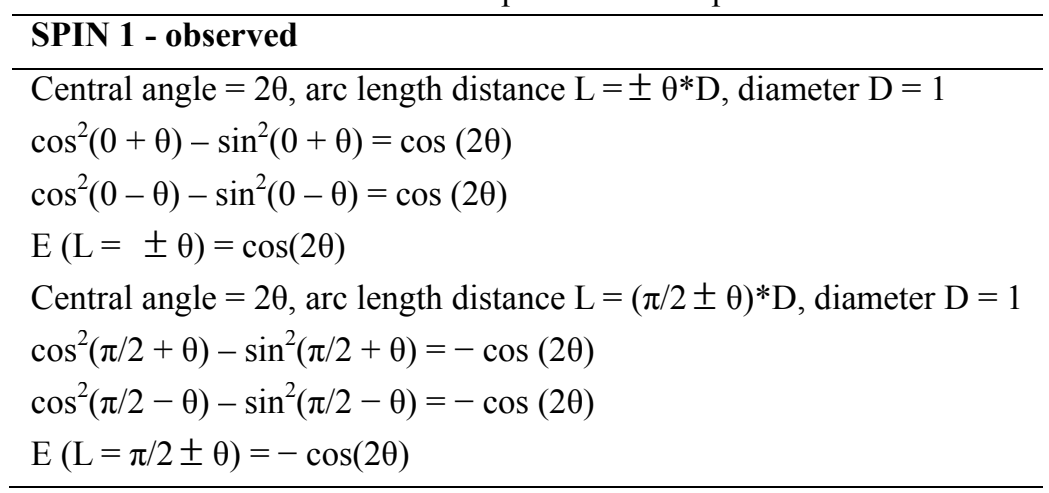


Spin $1 / 2$

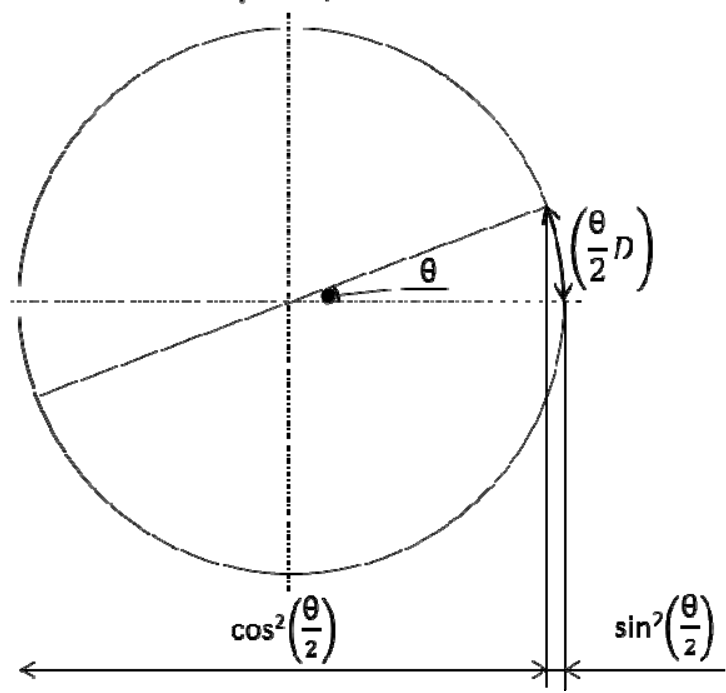

Spin $3 / 2$

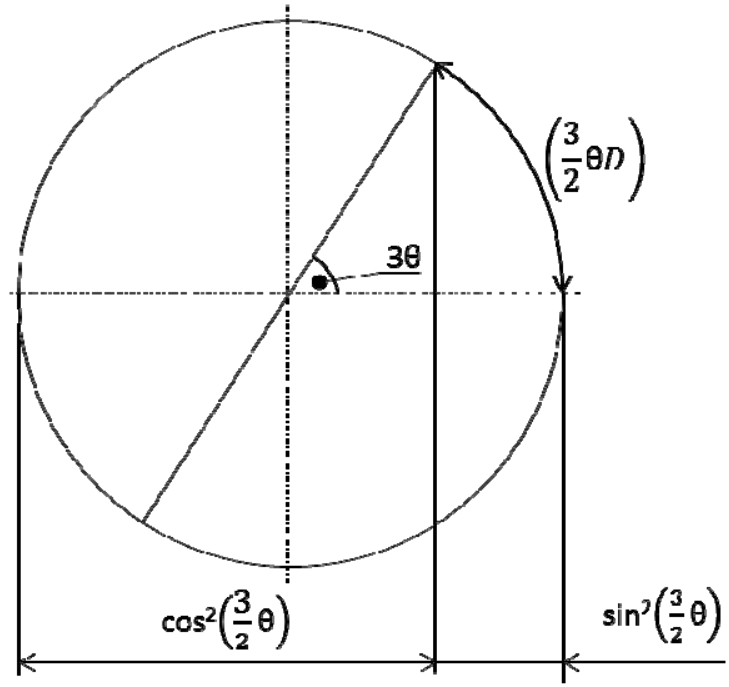

Spin 1

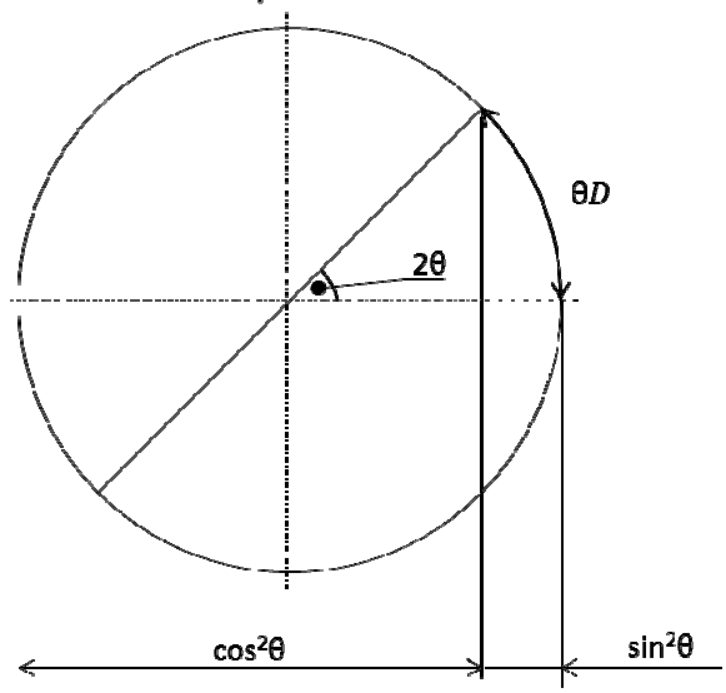

Spin 2

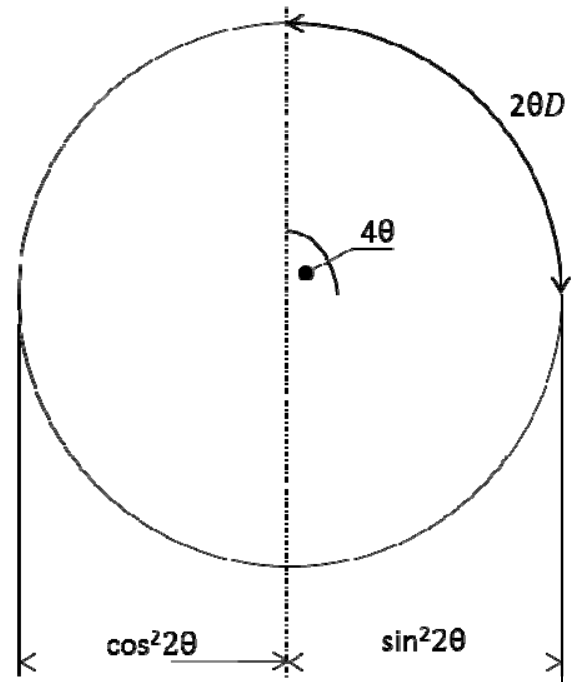

Figure 6. Dependence of the arc length distance for correlated particles on their spin

Table 9. Correlation and anti-correlation outcomes for particles with spin $3 / 2$

\begin{tabular}{l}
\hline SPIN 3/2 - predicted \\
\hline Central angle $=3 \theta$, arc length distance $\mathrm{L}= \pm 3 \theta / 2 * \mathrm{D}$, diameter $\mathrm{D}=1$ \\
$\cos ^{2}(0+3 \theta / 2)-\sin ^{2}(0+3 \theta / 2)=\cos (3 \theta)$ \\
$\cos ^{2}(0-3 \theta / 2)-\sin ^{2}(0-3 \theta / 2)=\cos (3 \theta)$ \\
$\mathrm{E}(\mathrm{L}= \pm 3 \theta / 2)=\cos (3 \theta)$ \\
Central angle $=3 \theta, \operatorname{arc}$ length distance $\mathrm{L}=(\pi / 2 \pm 3 \theta / 2) * \mathrm{D}$, diameter $\mathrm{D}=1$ \\
$\cos ^{2}(\pi / 2+3 \theta / 2)-\sin ^{2}(\pi / 2+3 \theta / 2)=-\cos (3 \theta)$ \\
$\cos ^{2}(\pi / 2-3 \theta / 2)-\sin ^{2}(\pi / 2-3 \theta / 2)=-\cos (3 \theta)$ \\
$\mathrm{E}(\mathrm{L}=\pi / 2 \pm 3 \theta / 2)=-\cos (3 \theta)$
\end{tabular}


Table 10. Correlation and anti-correlation outcomes for particles with spin 2

\begin{tabular}{l}
\hline SPIN 2 - predicted \\
\hline Central angle $=4 \theta$, arc length distance $\mathrm{L}= \pm 2 \theta^{*} \mathrm{D}$, diameter $\mathrm{D}=1$ \\
$\cos ^{2}(0+2 \theta)-\sin ^{2}(0+2 \theta)=\cos (4 \theta)$ \\
$\cos ^{2}(0-2 \theta)-\sin ^{2}(0-2 \theta)=\cos (4 \theta)$ \\
$\mathrm{E}(\mathrm{L}= \pm 2 \theta)=\cos (4 \theta)$ \\
Central angle $=4 \theta$, arc length distance $\mathrm{L}=(\pi / 2 \pm 2 \theta) * \mathrm{D}$, diameter $\mathrm{D}=1$ \\
$\cos ^{2}(\pi / 2+2 \theta)-\sin ^{2}(\pi / 2+2 \theta)=-\cos (4 \theta)$ \\
$\cos ^{2}(\pi / 2-2 \theta)-\sin ^{2}(\pi / 2-2 \theta)=-\cos (4 \theta)$ \\
$\mathrm{E}(\mathrm{L}=\pi / 2 \pm 2 \theta)=-\cos (4 \theta)$ \\
\hline
\end{tabular}

\section{Conclusions}

1) The ancient and medieval trigonometric functions Cosag $(2 \theta)=\operatorname{Cosagitta}(2 \theta)=\cos ^{2}(\theta)=\underline{\downarrow}$ and Sag $(2 \theta)=$ Sagitta $(2 \theta)=\sin ^{2}(\theta)=\uparrow$ were newly used as the mathematical tools for the description of correlation and anti-correlation outcomes of correlated photons.

2) The lengths Cosag $(2 \theta)=\cos ^{2}(\theta)$ and Sag $(2 \theta)=\sin ^{2}(\theta)$ are the heights of an arc in the circle with diameter $D$

$=1$ and the central angle $2 \theta$ and determine the probability of correlated and anti-correlated outcomes of correlated photons.

3) The arc length distance of polarization planes of correlated photons plays the significant role in their joint correlated and anti-correlated outcomes and can be experimentally modified by three half-wave plates (Alice's HWP, Bob's HWP, Claudius' HWP) - the tailor-made relative polarization of correlated photons (created by the SPDC Type I) can be achieved.

4) Claudius' Theorem based on the arc length distance of the polarization planes of correlated photons can describe and predict the correlation and anti-correlation outcomes for the correlated photons with the tailor-made relative polarization planes.

5) Spin of correlated particles (the arc length distance between the polarization planes in the circle with diameter $\mathrm{D}=1$ ) can be inserted into the Claudius' Theorem in order to calculate the correlation and anti-correlation outcomes for those correlated particles with spin $1 / 2,1,3 / 2,2$, etc.

\section{Acknowledgements}

This work was supported by the GMS Agency (Contract Number 69110/1992). We have found the valuable support on the web site www.wolframalpha.com with the corrections of the trigonometric functions.

\section{References}

Aspect, A. (2000). Bell's theorem: The naïve view of an experimentalist. Retrieved from http://arxiv.org/ftp/quant-ph/papers/0402/0402001.pdf

Aspect, A., Dalibard, J., \& Roger, G. (1982). Experimental test of Bell's inequalities using time-varying analyzers. Phys. Rev. Lett., 49, 1804-1807. http://dx.doi.org/10.1103/PhysRevLett.49.1804

Aspect, A., Grangier, P., \& Roger, G. (1982). Experimental realization of Einstein-Podolsky-Rosen-Bohm Gedankenexperiment: A new violation of Bell's inequalities. Phys. Rev. Lett., 49, 91-94. http://dx.doi.org/10.1103/PhysRevLett.49.91

Bell, J. S. (1964). On the Einstein-Podolsky-Rosen paradox. Physics, 1, 195-200.

Cabello, A. (2007). Bibliographic guide to the foundations of quantum mechanics and quantum information. Retrieved from http://www.johnboccio.com/research/quantum/qm_web/Bibliography.pdf

Clauser, J. F., \& Horne, M. A. (1974). Experimental consequences of objective local theories. Phys. Rev. D., 10, 526-535. http://dx.doi.org/10.1103/PhysRevD.10.526

Clauser, J. F., \& Shimony, A. (1978). Bell's theorem: Experimental tests and implications. Rep. Prog. Phys., 41, 1881-1927. http://dx.doi.org/10.1088/0034-4885/41/12/002

Clauser, J. F., Horne, M. A., Shimony, A., \& Holt, R. A. (1969). Proposed experiment to test local hidden-variable theories. Phys. Rev. Lett., 23, 880-884. http://dx.doi.org/10.1103/PhysRevLett.23.880 
Datta, B., \& Singh, A. N. (1983). Hindu Trigonometry. Indian Journal of History of Science, 18, 39-108.

Descartes, R. (1637). La Géométrie.

Einstein, A., Podolsky, B., \& Rosen, N. (1935). Can quantum-mechanical description of reality be considered complete? Phys. Rev., 47, 777-780. http://dx.doi.org/10.1103/PhysRev.47.777

Euclid, \& Heath, T. L. (1925). The thirteen books of Euclid's elements (2nd ed.). Cambridge: Cambridge University Press.

Fine, A. (1982). Hidden variables, joint probabilities, and the Bell inequalities. Phys. Rev. Lett., 48, 291-295. http://dx.doi.org/10.1103/PhysRevLett.48.291

Freedman, S. J., \& Clauser, J. F. (1972). Experimental test of local hidden-theories. Phys. Rev. Lett., 28, 938-941. http://dx.doi.org/10.1103/PhysRevLett. 28.938

Fry, E. S., \& Thomson, R. C. (1976). Experimental test of local hidden-variable theories. Phys. Rev. Lett., 37, 465-468. http://dx.doi.org/10.1103/PhysRevLett.37.475

Genovese, M. (2005). Research on hidden variable theories: A review of recent progresses. Phys. Rep., 413, 319. http://dx.doi.org/10.1016/j.physrep.2005.03.003

Giustina, M., Mech, A., Ramelow, S., Wittmann, B., Kofler, J., Beyer, J., .... Zeilinger, A. (2013). Bell violation using entangled photons without the fair-sampling assumption. Nature, 497, 227-230. http://dx.doi.org/10.1038/nature12012

Christensen, B. G., McCusker, K. T., Altepeter, J., Calkins, B., Gerrits, T., Lita, A. ... Kwiat, P. G. (2013). Detection-loophole-free test of quantum nonlocality, and applications. Arxiv: $1306.5772 \mathrm{v} 1$.

Kupková, E. (2008). Developing the radian concept: Understanding and the historical point of view. Quaderni di Ricerca in Didattica (Scienze Matematiche). 18, 73-85. Retrieved from http://math.unipa.it/ grim/Quad18_Kupkova_08.pdf

Kwiat, P. G., Waks, E., White, A. G., Appelbaum, I., \& Eberhard, P. H. (1999). Ultrabright source of polarization entangled photons. Phys. Rev. A., 60, 773-776. http://dx.doi.org/10.1103/PhysRevA.60.R773

Kwiat, P., Mattle, K., Weinfurter, H., \& Zeilinger, A. (1995). New high-intensity source of polarization-entangled photon pairs. Phys. Rev. Lett., 75, 4337-4341. http://dx.doi.org/10.1103/PhysRevLett.75.4337

Laloë, F. (2012). Do we really understand quantum mechanics? Cambridge: Cambridge University Press.

Maor, E. (1998). Trigonometric Delights. Princeton: Princeton University Press.

Marshall, T., Santos, E., \& Selleri, F. (1983). Local Realism has not been refuted by atomic cascade experiments. Phys. Rev. A., 98, 5-9. http://dx.doi.org/10.1016/0375-9601(83)90531-5

Matvievskaya, G. P. (1990). An outline of the history of trigonometry. Tashkent: Fan.

Ou, Z. Y., \& Mandel, L. (1988). Violation of Bell's inequality and classical probability in a two-photon correlation experiment. Phys. Rev. Lett., 61, 50-53. http://dx.doi.org/10.1103/PhysRevLett.61.50

Ptolemy, C. (1898). In Heiberg, J. L. (Ed.), Syntaxis mathematica. Leipzig: Teubner.

Rangarajan, R., Goggin, M., \& Kwiat, P. (2009). Optimizing type-I polarization-entangled photons. Opt. Expr., 17, 18920. http://dx.doi.org/10.1364/OE.17.018920

Rowe, M. A., Kielpinski, D., Meyer, V., Sackett, C. A., Itano, W. M., Monroe, C., \& Wineland, D. J. (2001). Experimental violation of a Bell's inequality with efficient detection. Nature, 409, 791-794. http://dx.doi.org/10.1038/35057215

Scheidl, T., Ursin, R., Fedrizzi, A., Ramelow, S., Ma, X. S., Herbst, T., ... Zeilinger, A. (2009). Feasibility of 300 $\mathrm{km}$ quantum key distribution with entangled states. New J. Phys., 11, 085002. http://dx.doi.org/10.1088/1367-2630/11/8/085002

Scheidl, T., Ursin, R., Kofler, J., Ramelow, S., Ma, X. S., Herbst, T., ... Zeilinger, A. (2010). Violation of local realism with freedom of choice. Proc. Natl. Acad. Sci. USA, 107, 19708-19713. http://dx.doi.org/10.1073/pnas.1002780107

Tapster, P. R., Rarity, J. G., \& Owens, P. C. M. (1994). Violation of Bell's inequality over $4 \mathrm{~km}$ of optical fiber. Phys. Rev. Lett., 73, 1923-1926. http://dx.doi.org/10.1103/PhysRevLett.73.1923 
Tittel, W., Brendel, J., Zbinden, H., \& Gisin, N. (1998). Violation of Bell inequalities by photons more than $10 \mathrm{~km}$ apart. Phys. Rev. Lett., 81, 3563-3566. http://dx.doi.org/10.1103/PhysRevLett.81.3563

Ursin, R., Tiefenbacher, F., Manderbach, T. S., Weier, H., Scheidl, T., Lindenthal, M., ... Zeilinger, A. (2007). Entanglement-based quantum communication over $144 \mathrm{~km} . \quad$ Nat. Phys., 3, 481-486. http://dx.doi.org/10.1038/nphys629

Van Brummelen, G. (2009). The mathematics of the Heavens and the Earth (The early history of trigonometry). Princeton: Princeton University Press.

Van Brummelen, G. (2013). Heavenly mathematics (The forgotten art of spherical trigonometry). Princeton: Princeton University Press.

Von Braunmühl, A. (1900). Vorlesungen über Geschichte der Trigonometrie. Leipzig: Teubner, 1900/1903.

Weihs, G., Jennewein, T., Simon, C., Weinfurter, H., \& Zeilinger, A. (1998). Violation of Bell's inequality under strict Einstein locality conditions. Phys. Rev. Lett., 81, $5039-5043$. http://dx.doi.org/10.1103/PhysRevLett.81.5039

White, A., James, D., Eberhard, P., \& Kwiat, P. (1999). Nonmaximally entangled states: production,

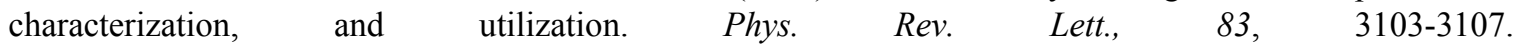
http://dx.doi.org/10.1103/PhysRevLett.83.3103

Wittmann, B., Ramelow, S., Steinlechner, F., Langford, N. K., Brunner, N., Wiseman, H. M., ... Zeilinger, A. (2013). Loophole-free Einstein-Podolsky-Rosen experiment via quantum steering. N. J. Phys., 14, 053030. http://dx.doi.org/10.1088/1367-2630/14/5/053030

Zeilinger, A. (1999). Experiment and the foundations of quantum physics. Review of Modern Physics, 71, S288-S297. http://dx.doi.org/10.1103/RevModPhys.71.S288

\section{Copyrights}

Copyright for this article is retained by the author(s), with first publication rights granted to the journal.

This is an open-access article distributed under the terms and conditions of the Creative Commons Attribution license (http://creativecommons.org/licenses/by/3.0/). 